\title{
Presenting a personalized mobile learning recommender system by using environmental and location information
}

\author{
Pooneh Seyedabolghasem, Akbar Khodaparast Haghi , Kamran Morovati \\ ${ }^{\prime}$ (Department of Information Technology The University of Guilan, Rasht, Iran, \\ ${ }^{2}$ (Department of Factually Engineering The University of Guilan , P.O.BOX 3756, Rasht, Iran, ) \\ ${ }^{3}$ Department of Computer Science University of Pune, PhD candidate in security, Pune, India
}

\begin{abstract}
The most important aims of mobile learning systems is providing relations between the real and virtual worlds and creating related instructing strategies with education activities in order to obtain appropriate educational achievements in addition to creating motivations in using mobile systems among the learning individuals. Offering potentials of instructing activities and places interested by individuals in this regard are among the most important aspects by which learners could increase their knowledge levels.

In this respect and by describing the case, we offer a model in the form of a mobile learning recommender system, abbreviated by "PMLES". Hence in this article we try to improve the performance of recommender systems in mobile learning environments by presenting an integrated system and providing a mobile learning recommender system, and along with that offer a learning method appropriate with the level of knowledge among different learners. The aim for this proposing model is increasing the performance rate of recommender systems by considering the educational conditions and developments of each learner in intercity environments.
\end{abstract}

Keywords: Mobile learning, personalization, recommender systems, environmental and location information

\section{INTRODUCTION}

The learning contents should be determined with appropriation to the person's needs and conditions, and on the basis of the goals for the learning system. Therefore, regarding the diverse applications of recommender systems and mobile learning, these systems could be used in environmental sciences and the matter could focus around the subjects including geology, mining, surveying, geography and other relevant topics due to non-urban environment in this proposed system. When, in a non-urban environment, a learner needs instruction information, the system should recommend proper instruction information to him/her that are appropriate with the attending place of that individual, by using allocating tools such as GPS and according to the moving direction and location of the person. It means that the learner should be within such a system and the instructions and suggestions to him/her should improve the learning trends of the person. The learning recommender system is presented in the first section. Then a definition of the features of PMLR system will be given. To follow, the modeling procedures of PMLRS and the states and architecture of this system will be considered. Afterwards, the PMLRS (personalized mobile learning recommender system) driver is described and finally, the obtained results are given.

\section{PERSONALIZED MOBILE LEARNING RECOMMENDER SYSTEM}

Constructivist theory and situated learning, as the two types of instruction theories should always be considered in learning recommender systems. The first theory expresses the notion that the learner always combines his/her knowledge and past experiences or understandings with his/her up-to-date activities and experiences to obtain new knowledge. Accordingly, the performance of the person is in trial and error basis and his/her feedback in each stage provides the possibility of obtaining the best output. The second theory is dealing with the surrounding world, sociability of individuals, social activities and obtained experiences or the acquired knowledge in that respect. It indicates that this theory is clearly related to the existing themes in the real world and their relevant problems. It is the physical attendance of people that enables them to enhance and reinforce their environmental information and learning about related subjects with the considered area.

The proposed combined recommender system method is established from combination of the methods based on the learners' behavioral patterns, using the recommender algorithms in classifying the learners, and also identifying the dependence of instruction contents with the environmental characteristics of features of education space in promoting the level of knowledge in learners. By dividing the learners into different groups, this system deals with considering the performance and behaviors of them and then recommends subjects or proper tests for them and regarding the obtained level of knowledge of each individual tries to offer the most appropriate educating place. The advantage of this method is in classification of the learners with regards to their level of knowledge. Hence after entering of a new learner, the system will be able to predict their functions 
before finishing their course of studies. Thus, the present system is divided into three general phases of definition of the characteristics, analysis of the architecture and modeling. Each phase will be herewith described.

\section{DEFINITION OF THE SYSTEM CHARACTERISTICS AND THE AIM FOR SYSTEM DEVELOPMENTS (PHASE I)}

According to the characteristics of mobile learning programs, the relevant benefits could be achieved by expressing some programs in executing learning activities outside the cities such as forests, pastures, mountains and training farms. Regarding the shortages observed in recommender and proposing systems and also the instruction topics in education domains, designing the optimized learning recommender systems seems to be essential for confronting the enormous rate of information and for instructing the learners. Hence in this article, an optimized recommender system is designed and proposed. To achieve the aims, first the relevant existences or components, features and assumptions related to the environment should be considered in order for a scheme to be proposed on its basis. To follow, the components and features of each part are to be dealt with.

\section{Components}

The parts and existing components of the systems should primarily be determined for designing PMLRS. These parts include: Users, mobile tools, global positioning system (GPS) and system database, each of which are described as follows:

\section{3-1 Users}

Here, the learner, as the user, is one of the most important components, having different appropriate characteristics with the existing definitions in the system and with different parts. A part of the data includes statistical information and primary information about the learner. This information includes:

Name, surname, age, sex, field of education, year, semester and the studied subjects, but another part of this information is related to the interests of the learner and includes the course, selected subject and the rate of enthusiasm to each topic and we enable the system to propose the studying subject and location via this information. Finally, the most important information is about the user's knowledge that is categorized into four criteria:

\section{1) Visual learning style criterion}

Regarding the environmental learning space, the qualitative level of audio-visual learning styles should be measured in individual learners in this system. Measurement of this criterion is done on the learners by using Felder-Silverman method that is "the best state of learning in audio-visual style is considering the visual, illustrative and environmental cases".

\section{2) Positioning potential criterion}

Another case to be measured in learners is their positioning capabilities in intercity environments such as education spaces. Hence we require measuring this criterion in learners and used SBSOD (Santa Barbara Sense of Direction) scale for its applications, since each individual could have the positioning capability to some extent according to his/her own environmental knowledge by this scale and the value of it could be measured.

\section{3) Intelligence and skills criterion}

Since talent is a relative measure in advancing an individual in an activity, if to acquire skills for an activity people are situated in similar conditions, they show differences with regards to the rate of acquiring those skills. Moreover, different people are different with each other for their intelligence levels and their situations depend on their intelligence that is measured along with the skills they have in a specific case. Thus we need to measure this criterion in the present education system to estimate the rate of developments in learners during their course of studies.

\section{4) Criterion for the level of knowledge}

The fourth and last feature to be acquired by the learners is the level of knowledge that these people have for their own selected subject, and the aim of this system is promoting this level during the course of studies. This part contains information about the considered subject of studies that the learner gains before starting the course and by attending in the class and accordingly, he/she should be able to respond to the questions given by the system to pass the course. 


\section{3-2 Mobile tools}

Due to mobility of PMLR System, the elaborating form between the system and the learners include mobile instruments such as tablets, I-pads and different types of cell phone devices. Before giving any definitions, it is assumed here that the used instruments in the system include all the system tools, since from one side, these instruments play an important role in exhibiting the contents and from another side, they could be used to determine the location of individuals.

But, regarding different characteristics in these devices such as the monitor, processing ability, place of restoring the data, method of connecting to the network, consuming power in batteries and other relevant features, recognizing the portable device used by the system is quite important. In analyzing the characteristics of these devices for their system behaviors, IMEI codes of identified cell phones could be used to be conformed to WURFL open text file that contains the information about different mobile devices with the updating potentials, for the system to recognize, during sending instructing contents, whether the user device supports audio-visual formats or it is only capable of receiving images.

\section{3-3 System Database}

To create an automation environment for management of education affairs and providing the potential for proposals in the present system we need user database, queries and instruction resources database, database under web, and location and environment information database.

\section{3-3 Characteristics of users, environment and activities}

To coordinate the inter-systematic subsistence with system definitions, we need to define the specificities for users, environment and existing activities in the system to be described as follows:

\section{1- Users' specifications}

Generally, the learners using PMLRS should have primarily enrolled in the system and by enrolling; they should consider the definitions of personal specifications, interests and their priorities. These specifications are as follows: Name, surname, age, sex, e-mail address, user's name and password and other related information. On the other hand, each learner registers primary information in the system according to his/her priorities and interests although changing some of this information is possible in next stages. The interests and priorities could include the points such as education, field of studies, passed courses, interested subjects and other required matters. All this information is registered in the users' profiles that are classified into two static and dynamic categories in accordance with the registered information. Static information is the user's specification that in most cases no changes are occurred in them, while the items like the user's priorities could be included in dynamic information, since it is possible for them to be changed by passage of time to be updated. For instance, when a person passes a credit in each semester, the number of his/her passes credits is increased. On the other hand, increasing the rate of knowledge of the individual could provide the change in some of his/her interests including specific topics or even some scientific non-academic subjects.

\section{2- Location and environmental specifications}

Since the selection of education environment could be based on the considered location and subject, the considered environmental specifications should be defined for the system to be able to identify the relevant location information. Due to the selected subject regarding intercity environments such as mountains, forests, pastures and farms as the target places, a region with diverse environmental specifications like variety of mountains, trees, stones, layers of earth, soils, plants, etc. should be considered for the present project to be stored in the relevant server as a GIS map.

Moreover, a description of the environmental specification for each of the above cases is attached to the considered section. After selecting the considered region and determination of the specifications of each section, different route(s) should be considered as the default on the graph for the information regions to be classified. The As a result, the moving position of each person could be classified into different sites in accordance with numerous information around him/her, for the relevant descriptions related to the existing specifications in the region to be defined. For instance a site could include mountains with different types of igneous stones, sedimentary stones and metamorphic rocks that each type could be observed in different layers of mountain sections.

\section{3- Activities specifications}

Among the most important parts in designing PMLRS is determination of executable activities for the learners, while in situated instructions, the aim is familiarizing the learners with the specifications of the surrounding environment and identifying the cases that promote the knowledge level in these people with respect to the relevant subjects. Accordingly, each learner should deal with executing activities such as 
responding to the questions, moving in right direction and proper use of previous knowledge, so that he/she could be able to do his/her course of studies.

The best way to help the students and simplicity in using the present system is installing such a system on most cell phones and mobile devices of individuals. Now by assuming the existence of mobile learning programs on cell phones and possibility of using them in defined places in the system and due to the system requirement to positioning and registration of the people's moving routes and comparing them with the right direction, the cell phones should be equipped with GPS receivers, in which case either the cell phone itself has such a facility or an external receiver should be connected to it. After this stage, the learner should try to move to the direction and have access to the existing learning materials in the environment by the information he/she has gained in the classroom.

Regarding the environment specifications, various questions should first be defined that are relevant to the selected subject and related scores should be added for the learners' responses to the questions. For example, some questions are asked from the person about different types of existing stones in the considered place and the person should be able to answer the questions according to his/her previous observations and knowledge. One of the important features for the questions in each section is the level of the question, which is categorized into five classifications; very simple, simple, moderate, hard and very hard. On the other hand, one of the most important activities in the present scheme is the movement of the person in the right direction to obtain the relevant instructions by going towards the whole route, from the start to the end. This by itself requires giving scores for the moving trends; from a point to the next. This is similar to a game that the person would be able to move on by going from a stage to the next stage and solve the required problems in each route.

\section{MODELING PERSONALIZED LEARNING RECOMMENDER SYSTEM (PHASE II)}

Presenting a model in accordance with the users' functions, interests and their environmental information is essential for personalizing the presented concepts and contexts by PMLRS, and this part could be considered as the central core of the system, since it is here that the required information should have coordination to provide the best offer.To facilitate this case, the system needs a clear understanding of the specifications of the learners and their elaborations with the system. These specifications include the existing data in the learners' profiles that consist of interests, skills, priorities, level of knowledge and the location of those learners that the system should conform itself with that, for personalizing its offering trends. To achieve this goal, we hereby deal with analyzing the architecture of PMLRS, make the models of the components and determine the method of performance of each model in the present learning recommender system through designing a combined model for recommender algorithms and providing the methods for learning the device.

\section{THE STATE OF PERFORMANCE AND ARCHITECTURE OF PMLRS}

One of the best states in designing mobile learning programs is implementing them on infrastructures of electronic learning systems. The reason for this is the existence of LMSs (learning management systems) and LOs (learning objects) as well as other existing databases in these systems that prevent extra costs and loss of time in such systems. Hence in designing PMLRS, it is better first to implement the systems infrastructures on the existing LMSs. Assuming that all the required databases for the system is existing, we hereby deal with the analysis of the architecture and operating trends of the target system.

After installing the considered program on the learner's mobile device and enrollments by him/her and defining the levels by the system, by activating the program in the next stages, the GPS receiver is activated and identifies the location of the person. Then, according to the selected course and subject at the enrollment stage by the learner and finding the start point by the system, the instruction trend and location are suggested with regards to the person's level of knowledge. The learner should also move in the right direction due to his/her own primary information. The recommender system is hereby proposing the considered instructing subjects. For instance, if the field of studies of the learner is geology, the required information based on geological specifications is presented to him/her by the system. Some questions are also asked from him/her about the same subject in the learning place for him/her to answer them. If the person could respond correctly, the nest learning objects are given and incase of incorrect answers, the recommender system provides the prerequisite information to him/her. Moreover, if the person is missing the proper direction, the system warns him/her and provides the correct direction. The verified direction is also considered by the recommender and the device algorithms. This trend continues up to the end of the instruction course. 


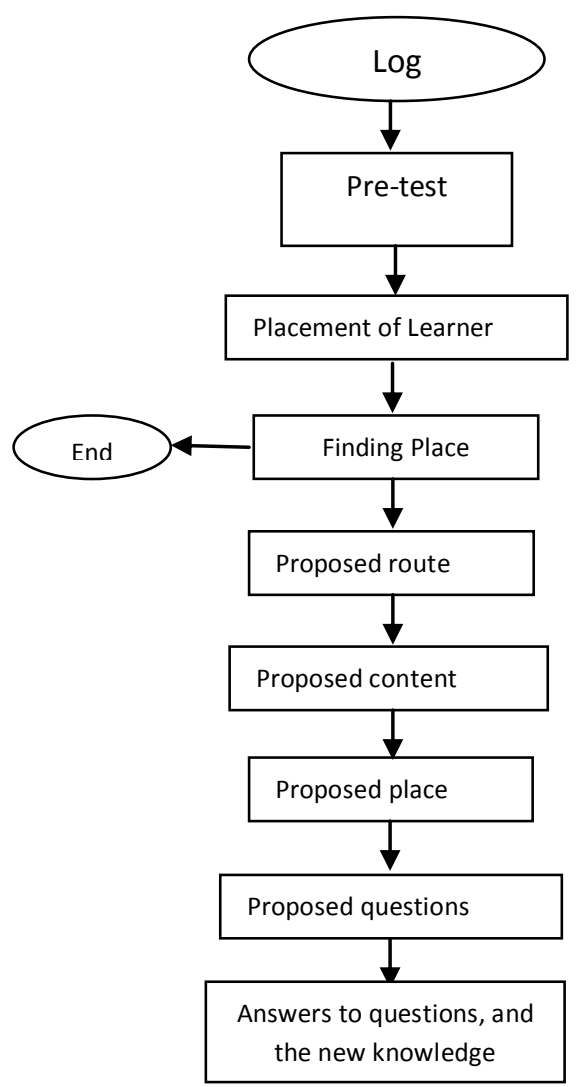

Figure (1): Outline of Performance Recommender Systems Quality (PMLRS)

\section{PMLRS ARCHITECTURE}

As the first step for the present learning recommender system, the learner should enroll in the system and provide primary information for it, for the system to obtain a default from the person's specifications and priorities. Moreover, the learner obtains a user name and password through enrollment that causes exclusiveness and security in the system. To provide relevant information, the learner should enroll via his/her cell phone or mobile device. This information is stored in a database that is considered for such matters to be used in next stages. After sending the request for registration, the information is considered in the server to be verified. Then, a message is sent to the system regarding the existence or non-existence of defects, and in case of verification, the information is stored in the server. In other case, a message regarding the enrollment error is sent to the program for the learner to correct the mistakes. After the enrollment and receiving user name and password, and installation of the program in the cell phone, the learner could have his/her connections by relevant LMS and LO. Whenever the learner attends the instruction (learning) camp, he/she will have the possibility to activate the considered program by entering the user name and password. By activating the program, the GPS receiver is activated and requires the location of the person from the transmitter. This request coordinates the existing default place in the spatial database and the obtained location from GPS satellites to verify the location. After verifying the location, the place of the person's attendance in the spatial database is registered in the system and his/her information domain is determined. Afterwards, an answer is sent in accordance with receiving the starting place of the person.

After the system is informed about the place and the surrounding area, it could offer the course, subject, routes and relevant places with regards to the selected course and subject by the learner. On the route, the system suggests the learning objects appropriate to the person's specifications. By the person's attendance to the learning place, the system provides some questions to be answered by using the recommender algorithms and the scientific level of the questions differs, depending on the system information from the person's specifications and priorities. Finally the questions are sent to the existing program on the learner's mobile device. After sending the answers by the learner to the program and transmitting it to the learning server and analysis of the results, the scientific level of the learner could be defined and the obtained results are sent to the recommender motor for this part to determine LO and relevant questions to the surrounding environment of the learner through the obtained information by the user and the existing environmental information including the data and specifications of geological levels in the GIS map. This trend is continued to the end. 


\section{PERFORMANCE OF THE RECOMMENDER MOTOR OF PMLR SYSTEM} (PHASE III)

The function of recommender could generally be described as follows. As observed in fig. (2), whenever a learner enters the system, the existing specifications are first considered in the person's profile and after classification and extraction of the considered information and identifying the location of the person and the state of his/her elaborations with the system, the recommender designs a model of the learner via relevant algorithms and proposes the most appropriate selection to him/her by his/her mobile device. It is hereby necessary to mention that the user's profile has an important role in all the stages of proposing and the recommender could find the best and most appropriate context that could be given to the user by relying on the registered stipulated information in it and by other existing information in the database.

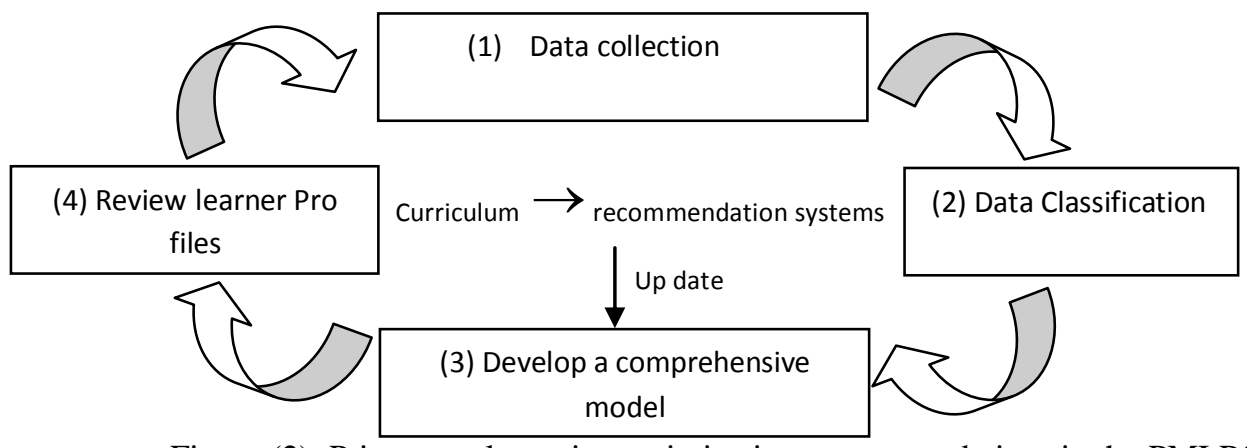

Figure (2): Private-cycle engine optimization recommendations in the PMLRS

By the first step of proposing, the system is only able to offer the most suitable selection or item to the learner in accordance with limited LO as the selected course and subject registered in the user's profile. After showing the presented suggestions by the recommender on the person's mobile set, he/she would indicate his/her interests to the proposal by accepting or refusing it and in case of acceptance of the proposal, the nearest or best route, with respect to the knowledge levels and the proposed course, will be recommended in combination with the requested course. In case of refusing the proposal, the system will introduce the most appropriate route to the learner with regards to the primary choice of the person. By passing the direction and receiving LO, appropriate with priorities and responses to the questions for each region, the learner continues his/her learning course and the system will alternatively also update the user's profile.

The important point is noticing the type of defined users by the system that is divided into two groups; newly arrived learners and the present learners of the course. The first group includes the people who had not entered the system by then and have only registered by giving specifications and who have answered the primary questions. The system could only predict the items according to the person's requests by relying on his/her given information. But in case of that the person passes this stage and starts the course, the considered learner is transformed into the present or existing user and after some more stages; the recommender could obtain more information about him/her and is able to provide better and more precise suggestions to him/her. To go through these stages the recommender needs to design a model of all the inter-system components and use recommending algorithms that could provide suggestions that are suitable with the considered matters.

\section{MODELING BY THE LEARNER}

The learner's model has always had direct relations with the user intermediate program and whenever the learner used the program, the system would collect the existing information, according to his/her functions and performance and designs a pattern with respect to the state of user's behavior and his/her actions.

As a whole, the main frame of PMLRS comprises of two parts: offline and online. In the offline section, the person provides the required information for the system for modeling by registering the primary information and the offline section for the learner provides the required information for the system by registering the primary information and answering the questions regarding intelligence quotient, visual learning styles, positioning and defining the knowledge level with respect to the selected course and subject. To prevent unnecessary connection between the server and the intermediate user, the existing program on the mobile set of the person consists of a small database to store the data in itself. On the other hand, by the learner entering the program, his/her positioning and timing situation is identified by the GPS and registered in that database. Then, the mobile learning program sends this information to the main system in the form of information packages for pre-processing and modeling. Afterwards, by entering the online section, the system could identify the learners' aims and behaviors by the created models, and accordingly present appropriate learning proposals by using recommender algorithms separately or in combined form, based on the contents including queries and answers, 
texts, images, sound and the route and location of each learner. Therefore the system required information is divided into primary information and in-learning information. In addition to the personal specifications such as name, surname and sex, the first category includes other key information such as the knowledge level and the learning style of the learners. But the in-learning information contains information such as places and routes, presented learning contexts and the new level of knowledge of learners and other demographic information that is registered implicitly in the system database. The related values to the demographic information for each group are considered as the weight for the modeling. The knowledge level of the learners in accordance with the selected subject among the primary information aspects is defined as the most important target for the system, and the three features of educational intelligence, visual learning style and the positioning potential of the individuals are also considered as the determining characteristics in identifying their behavior and the prerequisites for starting the learning process. A test is taken from the learner for weighing the first case and its score is registered as the primary entrance value. Some questionnaires are also given to each learner for the next three features and the scores for the answers are considered as weights. All the items are registered in the learning database or the learning management system in order to go into the next stages afterwards, by relying on the system information.

\section{GROUP MODELING OF THE LEARNERS}

After registering the primary information of the learners and determining the relevant model for each of them, the system could use recommender algorithms, such as collaborative filtering, based on content, statistics, community oriented and knowledge oriented aspects, to analyze the similarities between the learnersand use them for categorizing them in selecting the best possible states. We need two modeling levels for designing the group modeling of the learners. By using clustering methods, the similarities of the learners in their interests and enthusiasm towards the courses and going towards those routes and learning places and even the type of the contents that could be presented to each group are defined in the first level and hence, the relevant pattern could be extracted. It is while this is possible by different groups of clustering methods such as simple or multivariable "K-means" that are based on density and other existing cases. Then the system enters the 2nd level of modeling by the analysis and comparison of the clusters, and regarding the registered components, routes and learning objects in each cluster, the system will deal with extracting the relations to discover the dominating rules and these points are applied for modeling the group behavior in order to use the learners with the same clusters in grouping the newly arrived users, and use the existing regulations in each group in presenting the personalized offers.

Moreover, to prevent cheating among the learners with similar characters, the time of stating moving is different for each person, or different routes are proposed by the system. Hence after defining the first and starting place for the person by GPS of the cell phone and suggestion for the most suitable route, no other users would start from that point at that moment, warnings are given in case of the presence of any similar persons and another route would be proposed to the second learner. Anyhow, in case the learners possess different levels of knowledge, they will be allowed to start moving from the same point.

On the other side, regarding the precise scheduling in instruction and responding to the questions, individuals could not elaborate directly and could not exchange information during the course and it is only the system that provides learning objects and relevant questions on the routes to the education and training places, by using the acquired information from the learners.

\section{MODELING THE ENVIRONMENTAL DATA AND MODELING OF THE LEARNERS DURING THE COURSE}

The main part of this instruction system; or the environmental data is related to the appropriate places on the GIS map. The environmental data indicates instruction objects that are selected according to the course and the subject and based on the environmental characteristics of education region shave different levels of simplicity or hardness of the identifiable points in the area. To identify the environmental characteristics in each region by the learner, the regions consist of questions related to the existing conditions, according to their rate of hardness, and hence by the end of a one day course, the training course is divided into 20 general stages for promoting the knowledge levels in the learners. Moreover, regarding the classification of the knowledge levels in individuals that indicate the scores ranging from 1 to 10 , each knowledge level should be assigned to at least 20 different or similar places. Therefore, after calculating the level of each learner's knowledge, the places that could be proposed are introduced to him/her according to the classification. On the other hand, there is a possibility in each place that the environmental data is contrived with different hardness degree and thus the learner may be present at one place or pass through a route for more than one time. This could indicate the importance of some notions or the learner's enthusiasm towards some points.

According to this approach, there is the possibility of two types of clustering for environmental data; one type with regards to their rate of hardness and another, with respect to the number of attending times by the 
learners. Since as the default some learners with low knowledge levels require to pass the routes and attend at more places for learning, more places are offered to them by the beginning of their attendance in the system. Then, regarding their advancement during the course, the environmental data hardness criterion will be measured and other new routes will be offered to them.

\section{Modeling of the learners during the course}

After completing the basic information by the learner, the learning program requests him/her to start the course, at the next stage and by confirming or accepting that by the learner, the learner's location would be identified by GPS. In case of his/her presence in the training campus, the considered point is sent to the server to be regarded as the start of the course. Then by the first stage, the system identifies and offers the nearest or most appropriate point to the learner and simulates a model up to reaching the target point by the learner in accordance with his/her level of knowledge and his/her type of cell phone. Accordingly, the system identifies the executable learning objects in the learner's cell phone to be presented. By arriving the learner to the learning place, the learning objects are sent to the cell phone in multiple choice according to the received instructions, the hardness rate in understanding the items and identification of the environmental specifications by the learner, and he/she should answer the questions in due time and at each location. Here, according to the dependence of the knowledge level on the number of correct answers and the response time in the considered stage, the calculation and analysis method of the knowledge level will be different to the primary state, and we deal with it in the next section.

This instruction trend could improve the knowledge level of the learner in answering the questions and changes his/her knowledge level in each stage. It is here that the instruction trend is no longer dependent on the previous level of knowledge and a new level will replace that. The system proposes the new routes and learning places to the learner with regards to the new level of knowledge. By identification of the changes by the system, some changes are applied on the model of the learner and a new model is obtained with respect to the learner's behavior, through which the system requires to update the previous model and produce a new paradigm of the person's behavior with respect to his/her location and moving routes. This aspect indicates the dynamism of the present system that causes a better understanding about both, the present and newly arrived learners' behaviors.

\section{CALCULATION OF THE LEARNERS' KNOWLEDGE LEVELS}

To analyze the efficiency and effectiveness of any system, we should consider some parameters, and one of the most important aims in the present system is promoting the knowledge levels of the learners during the course. Since the environmental education campuses are for 14 day courses, the first day is usually considered for familiarity of the learners with regulations about the campus and the other 13 days could be used for instructions and promoting the knowledge level of the learners and since the present mobile learning system is an aided tool for the learning trends of the course, it is introduced to the learners and its functions are described to the participants in the first day. The amount of information, the knowledge level, and method of elaboration of people with the environment should then be determined at the same day and this is why the calculation of the knowledge levels in the group is potentially important for the system. Accordingly, the learners should make the system to recognize them by answering the considered question, for the rate of changes in them to be measureable and could be predicted, and the ascending or descending rate of knowledge in them could be discovered, so that relevant suggestions could be made. Therefore the learners should answer 30 scientific questions about their own field of studies in the first day, in an hour. The system, thence calculate the scores by registering the time and the number of correct answers to determine their primary knowledge levels, so that the possibility of finding the future knowledge levels to be provided. On the other hand, in accordance with the levels related to any point that are within the range of 1 to 10, the education and training regions considered in the area would take 5 questions, having the scores each of 5 out of 100, and the response time is considered to be 10 minutes for each stage. The learners will therefore be able to obtain the scores by going through the maximum of 20 stages per each day and along with it could promote their knowledge levels and achieve experiences related to their fields of studies. The more questions that are answered in the shortest time indicate the learner's higher level of knowledge that will cause his/her advancing to higher stages and levels.

\section{CONCLUSION}

To promote the learners' level of knowledge in intercity area, their most important characteristics and priorities are extracted by the system in the present project, to be stored in the database of the system. Then, regarding the selection made by the learner, various education /training places are offered. By going through this stage and answering the questions at each stage up to the end of the course, the learner will have the chance to have his/her knowledge level to be promoted. The advantages regarding the mobile learning characteristics could be taken into account by providing the programs in executing intercity learning activities, in places like 
forests, pastures and mountains. Moreover, the necessity of providing proposing instruction contexts and subjects in such areas could well indicate the need to design PMLR System.

\section{REFERENCES}

[1] J. Yin-Kim Yau, Ph.D thesis, in partial fulfilment of the requirements for the in Computer Science University of Warwick, Department of Computer Science, April 2011.

[2] E.Klobfer, Augmented Learning Research and Design of Mobile Educational Games , 2008.

[3] Piaget, J. The development of thought: Equilibration of cognitive structures, trans. A. Rosin. New York: Viking Press., 1977.

[4] V. Glasersfeld, E. 1995. A constructivist approach to teaching. In Constructivism in education, ed. L. P. Steffe and J. Gale, 3-15. Hillsdale, NJ: Erlbaum.

[5] Bruner, Jerome. Actual minds, possible worlds. Cambridge, MA: Harvard University Press. 1986.

[6] Lave, J., and E. Wenger. Situated learning: Legitimate peripheral participation. Cambridge: Cambridge University Press, 1991.

[7] Lave, J., and S. Chaiklin, eds. Understanding practice: Perspectives on activity and context. Cambridge: Cambridge University Press, 1993.

[8] Wenger, E. Communities of practice. learning, meaning, and identity. Cambridge: Cambridge University Press, 1998.

[9] Chen, Y. S., Kao, T. C., Yu, G. J. \& Sheu, J. P. A mobile butterfly-watching learning system for supporting independent learning, International Workshop on Mobile Technologies in Education, pp. 1118, 2004.

[10] R.M. Felder, Learning and Teaching Styles In Engineering Education, , North Carolina State University Linda K. Silverman, Institute for the Study of Advanced Development, 1998.

[11] M. Hegarty, Anthony E. Richardson, D. R. Montello, K. Lovelacea, I. Subbiah, Development of a selfreport measure of environmental spatial ability, Elsevier, March 2002.

[12] H.Pashaee, Theory and application of Intelligence and personality tests, Solhan Publications, Sixth edition, 2009.

[13] K. Verbert, N.Manouselis, X. Ochoa, M. Wolpers, H. Drachsler, I. Bosnic, E. Duval, Context-aware Recommender Systems for Learning: a Survey and Future Challenges, JOURNAL OF LATEX CLASS FILES, VOL. 6, NO. 1, JANUARY 2007. 